\title{
Background Parenchymal Enhancement in Contrast-enhanced Spectral Mammography: A Retrospective Analysis and a Pictorial Review of Clinical Cases
}

\author{
ROSARIA MEUCCI ${ }^{1,2}$, CHIARA ADRIANA PISTOLESE ${ }^{1}$, TOMMASO PERRETTA ${ }^{1}$, GIANLUCA VANNI ${ }^{2}$, \\ EMANUELA BENINATI ${ }^{1}$, FEDERICA DI TOSTO $^{1}$, MARIA LINA SERIO $^{1}$, AURELIA CALIANDRO $^{1}$, \\ MARCO MATERAZZO ${ }^{2}$, MARCO PELLICCIARO ${ }^{2}$ and ORESTE CLAUDIO BUONOMO ${ }^{2}$ \\ ${ }^{1}$ UOC Diagnostic Imaging, Policlinico Tor Vergata University, Rome, Italy; \\ ${ }^{2}$ Breast Unit, Department of Surgical Science, Policlinico Tor Vergata University, Rome, Italy
}

\begin{abstract}
Background/Aim: Despite the popularity of contrast enhanced spectral mammography (CESM), univocal classification of the background parenchymal enhancement (BPE), a bilateral enhancement of the normal breast parenchyma after contrast administration, is lacking. The present study aimed to evaluate the application of BPE Breast Imaging Reporting and Data System Magnetic Resonance (BI-RADS-MR) score for the CESM BPE. Moreover, a pictorial review of four different cases with CESM is provided. Patients and Methods: A single-center, retrospective study from a prospectively maintained database of all women undergoing digital mammography (DM) and CESM in our institution between 2016 and 2019. DM and CESM were classified by two experienced radiologists. Results: No statistically significant difference between DM breast density and BPE CESM classification was found. Agreement between readers ranged from substantial to almost perfect. Conclusion: BIRADS-RM score for the CESM $B P E$ represents a handy option for radiologists with high inter-reader and DM agreement.
\end{abstract}

Breast cancer (BC) is the leading cause of neoplasm affecting 2.1 million women per year worldwide (1). Despite the epidemiologic burden, deep knowledge of cancer

This article is freely accessible online.

Correspondence to: Marco Materazzo, Breast Unit, Department of Surgical Science, PTV: Policlinico Tor Vergata University, Viale Oxford 81, 00133 Rome, Italy. Tel: +39 3395685883, e-mail: marco.materazzzo@ptvonline.it

Key Words: Contrast enhanced spectral mammography, background parenchymal enhancement, breast imaging-reporting and data system, breast cancer, breast glandular density. biology, early detection and reduction of surgical impact have provided steady improvement in long term outcomes in the recent years (2-4).

In Europe, early detection has been provided through mammographic screening programs, which alone provided reduction in morbidity related with locally advanced breast cancer (LABC) diagnosis, and cancer-specific mortality between $12 \%$ and $58 \%(5,6)$, with reduction of locoregional lymph nodes involved $(7,8)$ and distant metastasis $(9,10)$.

However, during screening when mammography is not sufficient, additional second level imaging such as digital breast tomosynthesis, ultrasound, magnetic resonance (MR) and/or contrast enhanced spectral mammography (CESM) are required (11).

Within the last few years, CESM gained popularity as a diagnostic technique for $\mathrm{BC}$ detection. When compared with breast MR, CESM is a faster and cheaper technique, which is characterized by greater patients' comfort (12). Additionally, it has also been proven that CESM has a very low learning curve for imaging specialists (13).

Thanks to these advantage, current CESM indication shifted from second level imaging alternative to MR in case of contraindication (e.g., patients with metallic prosthesis or claustrophobic) to evaluate inconclusive findings on mammography, and assess breast symptoms, cancer staging, and the response to neoadjuvant chemotherapy $(12,14)$. Moreover, a recent use as an alternative examination to MR for high-risk screening has also been reported (13-15).

Regarding the method, CESM can be performed using two different techniques, temporal subtraction (between pre- and post-contrast acquisition) and dual-energy subtraction. The latter one combines an iodinated contrast agent with dualenergy subtraction technique, consisting of a pair of lowenergy and high-energy images acquired after contrast administration and used to construct the final combined image (16). 
Contrast agent administration, which is selectively concentrated in the tumor bed due to tumor tissue neoangiogenesis, provides better neoplasm visualization compared to the traditional mammography (12).

However, CESM limitations consist of artefacts in patients with breast implants $(17,18)$, the radiation dose associated with CESM, and the background parenchymal enhancement (BPE) phenomenon. BPE, previously described in RM, is a bilateral enhancement of the normal breast parenchyma after contrast administration, depending on tissue venous blood pool and its permeability to the contrast agent.

Despite the fact that the BPE phenomenon has already been studied and higher grade BPE has been associated with higher risk of BC (16), univocal BPE in CESM classification is lacking. Therefore, the aim of the present study was to assess a proposal of classification of BPE in CESM and a pictorial review of our case series.

\section{Patients and Methods}

Study design and patient selection. A single-center, retrospective study from a prospectively maintained database was designed. The local Institutional Review Board waived the need of a formal approval due to the retrospective design. Patients with higher risk of $\mathrm{BC}$ development according to the Gail Score ( $>15 \%$ lifetime risk) (19) were enrolled in our high risk screening program with mammography and CESM between 2016 and 2019. Before admission in the high-risk screening program, our patients routinely sign written informed consent for retrospective study. If digital mammography (DM) or CESM was not performed in our facility, patients were excluded from the analysis.

All exams were blindly evaluated by two experienced radiologists with 15 years of interpretation experience in mammography and five years in CESM, who are not routinely involved in our high-risk screening program.

DM was graded separately according to the breast imaging reporting and data system (BI-RADS) density classification as predominantly fatty (BIRADS 1), scattered fibroglandular (BIRADS 2), heterogeneously dense (BIRADS 3), or extremely dense (BIRADS 4) (20).

After evaluation of DM, blind evaluation of CESM was performed. BPE in CESM was firstly defined as absent or present. When BPE CESM was detected, BPE was classified as BI-RADSRM score according to localization (symmetric or asymmetric) and amount [absent, grade 1 (minimal), grade 2 (mild), grade 3 (moderate) or grade 4 (marked)] (21).

When radiologists assigned different scores in DM evaluation, collegial discussions were performed in order to obtain unique CESM BPE classification and unique BPE CESM evaluation.

CESM protocol. In our clinical practice, DM is routinely performed with GE Healthcare Dual Energy Mammography System (Milan, Italy). Moreover, our high-risk screening protocol of CESM was performed with GE Healthcare Dual Energy Mammography System after intravenous administration of $1.5 \mathrm{ml}$ of iohexol Omnipaque 350 (GE, Shanghai, PR China) per kilogram of body weight at an injection rate of $3 \mathrm{ml} / \mathrm{s}$. Once the injection was completed, the patient was positioned to acquire the first mammographic image, which was obtained approximately 2.5 minutes after injection. All four images (craniocaudal and mediolateral oblique images of each breast) were obtained within 5 minutes. For each view, a low-energy exposure (26-30 kVp) and a high-energy exposure (45-49 kVp) were acquired. A recombination algorithm was used to subtract the unenhanced breast tissue and provided a subtracted image on which areas of contrast enhancement were highlighted. The low-energy exposure images were used to determine breast density. All four views were used for BPE evaluation.

Statistical analysis. All data were recorded onto an EXCEL database (Microsoft, Redmond, WA, USA). Dummy variables reported as numbers and percentages: Chi-squared and Fisher's exact tests for statistical significance were performed. Variables with a $p$-value $<0.05$ were considered statistically significant.

A $\mathrm{K}$ coefficient was calculated to assess pairwise agreement between each pairing of the two readers for categorization of BPE and assessment of overall agreement; the mean $\mathrm{K}$ value was calculated from these pairs. Strength of $\mathrm{K}$ agreement was defined as less than 0.00, poor; 0.00-0.20, slight; 0.21-0.40, fair; 0.41-0.60, moderate; 0.61-0.80, substantial; and 0.81-1.00, almost perfect.

\section{Results}

Among 170 patients, who performed CESM in the study period, 15 were excluded: five patients performed CESM as assessment of neoadjuvant chemotherapy response and 10 patients performed DM and CESM as postoperative followup. Consequently, the final cohort consisted of 155 patients.

Regarding the DM pattern, 65/155 (42\%) were classified in BIRADS 1, 28/155 (18\%) in BIRADS 2, 51/155 (33\%) in BIRADS 3, and 11/155 (7\%) in BIRADS 4. CESM BPE showed that 38/155 (25\%) were BPE absent, 55/155 (35\%) BPE grade 1, 30/155 (20\%) BPE grade 2, 22/155 (14\%) BPE grade 3, and 10/155 (6\%) BPE grade 4. No statistically significant difference between DM breast density and CESM BPE was found $(p>0.05)$.

Overall agreement for categorization of BPE among the readers ranged from substantial to almost perfect $(\mathrm{K}=0.60$ 0.96 ) with a mean $\mathrm{K}$ value of 0.743 . As pictorial review, we present 4 examples of different BPE.

\section{Clinical cases.}

Case No 1: Contrast-enhanced spectral mammography characterized by minimal BPE. A 59-year-old woman was called back to our institute to perform CESM in order to investigate a palpable lump, located at the upper outer quadrant of the left breast, neither identified by DM nor by ultrasound examination (US). Breast composition, defined by the visually estimated content of fibro-glandular dense tissue was classified as BI-RADS C.

Mammography (MG) showed a micronodular glandular pattern, spread bilaterally. Cranio-caudal (CC) and mediolateral oblique (MLO) CESM projections didn't show focal contrast enhancement bilaterally and documented a minimal BPE (Figure 1). 


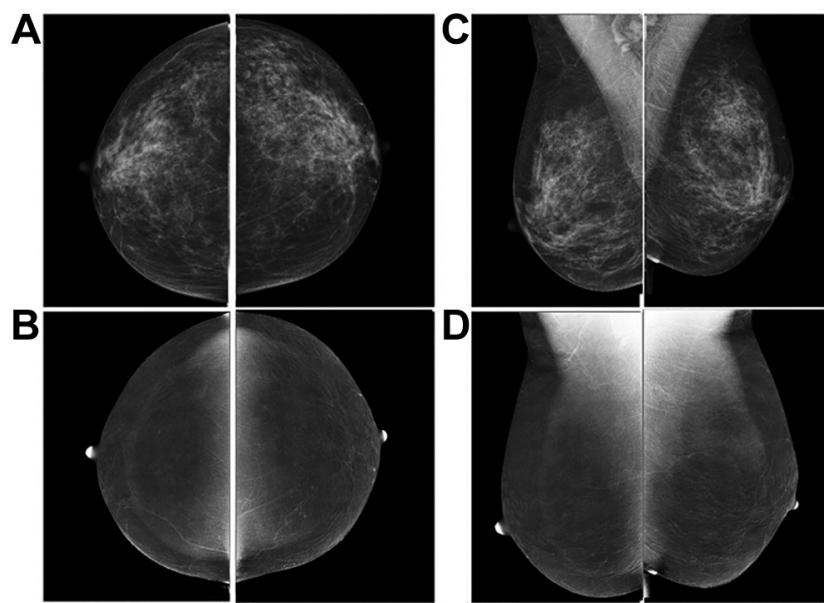

Figure 1. Case 1: 59-year-old woman DM and CESM with palpable lump in the upper outer quadrant of the left breast; DM and CESM. A, $C$ : $C C$ and MLO DM of fibro-glandular density tissue (BI-RADS C) B, $D$ : CC and MLO CESM projections with minimal BPE (grade 1). DM: Digital mammography; BI-RADS: breast imaging-reporting and data system; BPE: background parenchymal enhancement; CC: craniocaudal projection; $M L O$ : mediolateral oblique projection; CESM: contrast enhanced spectral mammography.

Case No 2: Contrast-enhanced spectral mammography characterized by mild BPE. A 62-year-old woman performed a CESM because of high familiarity risk. She was claustrophobic so she refused MR. Past medical history reported transthoracic repair of Morgagni's hernia (22). DM breast density was graded as high BI-RADS C. CESM didn't show any pathologic findings; small scattered areas of enhancement attributed to a mild CESM BPE were identified (Figure 2).

Case No 3: Contrast-enhanced spectral mammography characterized by moderate BPE. A 54-year-old woman performed a CESM as a diagnostic integration DM and US. Past medical history included kidney transplant in 2015, one abortion (23), and percutaneous coronary intervention (PTCA) after non-ST elevation myocardial infarction (NSTEMI) (24). Moreover, the patient reported irregular menstrual cycles and secretions from the left nipple. The patient's breast density DM was defined as high (BI-RADS $\mathrm{C} / \mathrm{D})$. CESM showed a widespread progressive and persistent enhancement in absence of focal pathological enhancement. These imaging features were compatible with moderate CESM BPE (Figure 3).

Case No 4: Contrast-enhanced spectral mammography characterized by marked BPE. A 48-year-old woman performed a CESM as a diagnostic integration DM and US. Patient's past medical history included Hepatitis C treated using a direct-acting antiviral (DAA) drug (25). The patient's
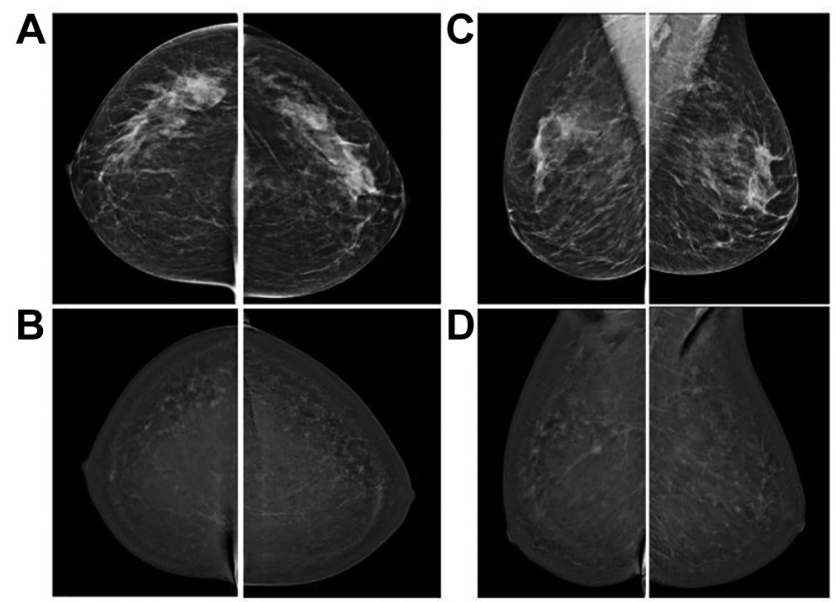

Figure 2. Case 2: 62-year-old woman high risk screening for BC; DM and CESM. A, C: CC and MLO digital mammography of fibroglandular density tissue (BI-RADS C) B, D: CC and MLO CESM projections with mild BPE (grade 2). BC: Breast cancer; BI-RADS: breast imaging-reporting and data system; BPE: background parenchymal enhancement; CC: craniocaudal projection; DM: digital mammography; MLO: mediolateral oblique projection; CESM: contrast enhanced spectral mammography.
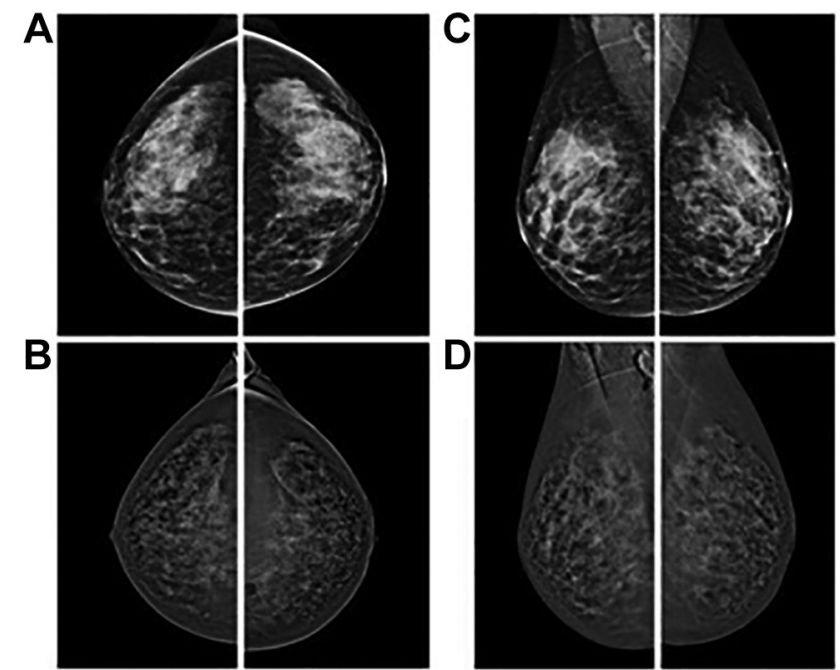

Figure 3. Case 3: 54-year-old II level CESM as II level imaging after $D M$ and US; DM and CESM. A, C: CC and MLO digital mammography of high-density tissue (BI-RADS C-D). B, D: CC and MLO CESM projections with moderate BPE (grade 3). BI-RADS: Breast imagingreporting and data system; BPE: background parenchymal enhancement; CC: craniocaudal projection; DM: digital mammography; MLO: mediolateral oblique projection; $M L O$ : mediolateral oblique projection; US: ultrasound; CESM: contrast enhanced spectral mammography.

breast density was high BI-RADS D. Because to the familiar risk, the patient was enrolled in our BC screening program with CESM. 


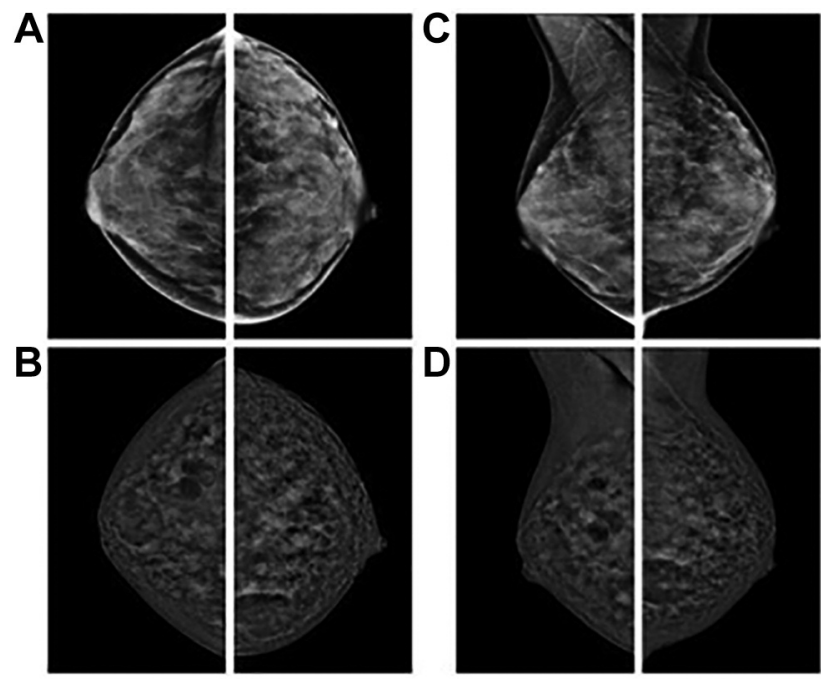

Figure 4. 48-year-old II level CESM as II level imaging after DM and US; DM and CESM. A, C: CC and MLO digital mammography of high breast density tissue (BI-RADS D). B, D: CC and MLO CESM projections with marked BPE (grade 4). BI-RADS: Breast imaging-reporting and data system; BPE: background parenchymal enhancement; CC: craniocaudal projection; DM: digital mammography; MLO: mediolateral oblique projection; US: ultrasound contrast; CESM: contrast enhanced spectral mammography.

CESM showed multiple focal areas of early and persistent enhancement, with micro and macronodular patterns, spread bilaterally across all quadrants. These imaging features were compatible with marked CESM BPE (Figure 4).

\section{Discussion}

CESM, firstly introduced in 2003 as alternative techniques to $\mathrm{MR}$, is currently gaining popularity thanks to its feasibility, compliance of patients, and shorter procedural time (12). When compared with breast MR, CESM has higher specificity and negative predictive value (NPV) $(100 \%)$, but a lower sensitivity $(88 \%)$ and a positive predictive value (PPV) of $76 \%$ (16).

Because to the shorter learning curve (13), many authors have proposed this imaging method as a solving tool for inconclusive findings on screening mammography, which is limited by the glandular density. Moreover, concerning cancer staging, CESM has repeatedly shown a comparable sensitivity value but a higher specificity compared to MR, which nowadays represents the gold standard $(13,16,26)$. Despite this popularity, due to the novelty of the procedure, standardized lexicon, recommendations, and classification are missing in CESM, as for CESM BPE. Moreover, after the first wave of COVID-19 pandemic $(27,28)$, unification of the classifications of the CESM technique is even more urgent to provide a faster approach, with a potential reduction in the time spent in hospitals by patients and an access of a greater number of patients to diagnostic procedures $(29,30)$.

BPE, the bilateral enhancement of the normal breast parenchyma after contrast administration, was firstly described in MR. Higher grade of BPE has been related to many factors such as radiotherapy and breast density, and hormone levels (26). Other factors linked to higher grade of $\mathrm{BPE}$ are the phase of menstrual cycle for women in the reproductive age, premenopausal status, and hormonal therapy (31). For this reason, some authors recommend performing MR between days 7-14 to minimize the masking effect in cancer detection (32).

Recently Sogani et al. showed that CESM BPE is comparable to MR BPE and is influenced in the same way by different and already known factors; moreover they reported a moderate intra-reader and inter-reader agreement (31). Furthermore, BPE is an important imaging aspect to evaluate, considering that it has been demonstrated to be an independent predictor of breast cancer risk, proportional to its grading. CESM BPE could be useful as an additional risk factor to detect women who need close surveillance. In a recent analysis, CESM BPE was more strongly correlated to cancer risk than other known risk factors, such as mammographic breast density, MR BPE, and fibroglandular volume (32).

As mentioned before, a common CESM BPE lexicon is still lacking and some authors suggested to use BIRADS-RM score for the MR BPE, with a visual score of the enhancement volume (minimal, mild, moderate, marked) and of its symmetric or asymmetric localization (21). In the present study, BIRADS-RM score for the CESM BPE represented a handy option for radiologists with high intraand inter-reader agreement and early experience in CESM.

We are aware that our research has some limitations. First, the retrospective and monocentric design may have influenced our results and no recommendations in clinical practice may rise from this analysis. However, the prospectively maintained database and the blind revision may have led to a reduction in this bias. In our pictorial essay, through a review of our clinical cases and a retrospective analysis, we proposed four different stages of CESM BPE from minimal to marked, using the dual-energy subtraction technique. Larger prospective trials may provide useful information on the role of BPE in CESM in terms of clinical outcome.

The state of art is unavoidable considering the emerging importance of this technique in breast cancer diagnosis and follow-up, based on the recent proof that CESM has similar sensitivity to RM and the higher specificity (16).

We consider it worthwhile that future radiologists get increasingly involved with CESM and its correlate imaging tools, as the BPE. This represents one of the most interesting and emerging approaches to evaluate in CESM exams. 


\section{Conflicts of Interest}

The Authors declare no conflicts of interest regarding this study.

\section{Authors' Contributions}

Study conception and design: Rosaria Meucci and Chiara Adriana Pistolese; Acquisition of data: Tommaso Perretta and Emanuela Beninati; Analysis of data: Federica Di Tosto and Maria Lina Serio; Interpretation of data: Gianluca Vanni, Meucci Rosaria, Perretta Tommaso; Drafting of article: Materazzo Marco, Buonomo Oreste Claudio and Rosaria Meucci; Critical revision: Vanni Gianluca and Marco Pellicciaro; Critical revision of literature: Marco Pellicciaro, Aurelia Caliandro, Rosaria Meucci and Marco Materazzo. Clinical Case data acquisition: Emanuela Beninati, Federica Di Tosto, Maria Lina Serio, and Aurelia Caliandro. All the Authors read and approved the final version of the manuscript.

\section{Acknowledgements}

The work was partially financially supported by the Italian Ministry of Health, Rome, Italy.

\section{References}

1 Sung H, Ferlay J, Siegel RL, Laversanne M, Soerjomataram I, Jemal A and Bray F: Global cancer statistics 2020: GLOBOCAN estimates of incidence and mortality worldwide for 36 cancers in 185 countries. CA Cancer J Clin 71(3): 209-249, 2021. PMID: 33538338. DOI: $10.3322 /$ caac. 21660

2 Associazione Italiana di Oncologia Medica: I Numeri del Cancro in Italia, 2019. Available at: https://www.aiom.it/wp-content/ uploads/2019/09/2019_Numeri_Cancro-operatori-web.pdf [Last accessed on December 18, 2021]

3 Vanni G, Materazzo M, Perretta T, Meucci R, Anemona L, Buonomo C, Dauri M, Granai AV, Rho M, Ingallinella S, Tacconi F, Ambrogi V, Chiaravalloti A, Schillaci O, Petrella G and Buonomo OC: Impact of awake breast cancer surgery on postoperative lymphocyte responses. In Vivo 33(6): 1879-1884, 2019. PMID: 31662515. DOI: 10.21873/invivo.11681

4 Roselli M, Guadagni F, Buonomo O, Belardi A, Ferroni P, Diodati A, Anselmi D, Cipriani C, Casciani CU, Greiner J and Schlom J: Tumor markers as targets for selective diagnostic and therapeutic procedures. Anticancer Res 16(4B): 2187-2192, 1996. PMID: 8694541

5 Zielonke N, Gini A, Jansen EEL, Anttila A, Segnan N, Ponti A, Veerus P, de Koning HJ, van Ravesteyn NT, Heijnsdijk EAM and EU-TOPIA consortium: Evidence for reducing cancerspecific mortality due to screening for breast cancer in Europe: A systematic review. Eur J Cancer 127: 191-206, 2020. PMID: 31932175. DOI: 10.1016/j.ejca.2019.12.010

6 Buonomo O, Cabassi A, Guadagni F, Piazza A, Felici A, Piccirillo R, Atzei GP, Cipriani C, Schiaroli S, Mariotti S, Guazzaroni MN, Cossu E, Simonetti G, Pernazza E, Casciani $\mathrm{CU}$ and Roselli M: Radioguided-surgery of early breast lesions. Anticancer Res 21(3C): 2091-2097, 2001. PMID: 11501831.

7 Van Zee KJ, Manasseh DM, Bevilacqua JL, Boolbol SK, Fey JV, Tan LK, Borgen PI, Cody HS 3rd and Kattan MW: A nomogram for predicting the likelihood of additional nodal metastases in breast cancer patients with a positive sentinel node biopsy. Ann Surg Oncol 10(10): 1140-1151, 2003. PMID: 14654469. DOI: 10.1245/aso.2003.03.015

8 Ielpo B, Pernaute AS, Elia S, Buonomo OC, Valladares LD, Aguirre EP, Petrella G and Garcia AT: Impact of number and site of lymph node invasion on survival of adenocarcinoma of esophagogastric junction. Interact Cardiovasc Thorac Surg 10(5): 704-708, 2010. PMID: 20154347. DOI: 10.1510/icvts.2009.222778

9 Buonomo OC, Caredda E, Portarena I, Vanni G, Orlandi A, Bagni C, Petrella G, Palombi L and Orsaria P: New insights into the metastatic behavior after breast cancer surgery, according to well-established clinicopathological variables and molecular subtypes. PLoS One 12(9): e0184680, 2017. PMID: 28922402. DOI: 10.1371 /journal.pone .0184680

10 Ielpo B, Mazzetti C, Venditti D, Buonomo O and Petrella G: A case of metachronous splenic metastasis from renal cell carcinoma after 14 years. Int J Surg 8(5): 353-355, 2010. PMID: 20438874. DOI: 10.1016/j.ijsu.2010.04.006

11 Niell BL, Freer PE, Weinfurtner RJ, Arleo EK and Drukteinis JS: Screening for breast cancer. Radiol Clin North Am 55(6): 1145-1162, 2017. PMID: 28991557. DOI: 10.1016/j.rcl. 2017.06.004

12 James JJ and Tennant SL: Contrast-enhanced spectral mammography (CESM). Clin Radiol 73(8): 715-723, 2018. PMID: 29937340. DOI: 10.1016/j.crad.2018.05.005

13 Lalji UC, Houben IP, Prevos R, Gommers S, van Goethem M, Vanwetswinkel S, Pijnappel R, Steeman R, Frotscher C, Mok W, Nelemans P, Smidt ML, Beets-Tan RG, Wildberger JE and Lobbes MB: Contrast-enhanced spectral mammography in recalls from the Dutch breast cancer screening program: validation of results in a large multireader, multicase study. Eur Radiol 26(12): 4371-4379, 2016. PMID: 27097789. DOI: $10.1007 / \mathrm{s} 00330-016-4336-0$

14 Tennant SL, James JJ, Cornford EJ, Chen Y, Burrell HC, Hamilton LJ and Girio-Fragkoulakis C: Contrast-enhanced spectral mammography improves diagnostic accuracy in the symptomatic setting. Clin Radiol 71(11): 1148-1155, 2016. PMID: 27296475. DOI: 10.1016/j.crad.2016.05.009

15 Alexander S, Dulku G, Hashoul S and Taylor DB: Practical uses of contrast-enhanced spectral mammography in daily work: A pictorial review. J Med Imaging Radiat Oncol 63(4): 473-478, 2019. PMID: 31332941. DOI: 10.1111/1754-9485.12927

16 Patel BK, Lobbes MBI and Lewin J: Contrast enhanced spectral mammography: a review. Semin Ultrasound CT MR 39(1): 7079, 2018. PMID: 29317041. DOI: 10.1053/j.sult.2017.08.005

17 Buonomo OC, Morando L, Materazzo M, Vanni G, Pistilli G, Palla L, Di Pasquali C and Petrella G: Comparison of round smooth and shaped micro-textured implants in terms of quality of life and aesthetic outcomes in women undergoing breast reconstruction: a single-centre prospective study. Updates Surg 72(2): 537-546, 2020. PMID: 32062785. DOI: 10.1007/s13304020-00721-w

18 Nori J, Gill MK, Vignoli C, Bicchierai G, De Benedetto D, Di Naro F, Vanzi E, Boeri C and Miele V: Artefacts in contrast enhanced digital mammography: how can they affect diagnostic image quality and confuse clinical diagnosis? Insights Imaging 11(1): 16, 2020. PMID: 32034578. DOI: 10.1186/s13244-0190811-x

19 Gail MH, Brinton LA, Byar DP, Corle DK, Green SB, Schairer $\mathrm{C}$ and Mulvihill JJ: Projecting individualized probabilities of 
developing breast cancer for white females who are being examined annually. J Natl Cancer Inst 81(24): 1879-1886, 1989. PMID: 2593165. DOI: 10.1093/jnci/81.24.1879

20 American College of Radiology, BI-RADS Committee, Morris EA, Comstock CE, Lee $\mathrm{CH}$, Lehman CD, Ikeda DM and Newstead GM: ACR BI-RADS ${ }^{\circledR}$ atlas, breast imaging reporting and data system. $5^{\text {th }}$ Edition. Reston, VA, USA, American College of Radiology, 2013.

21 Rao AA, Feneis J, Lalonde C and Ojeda-Fournier H: A pictorial review of changes in the BI-RADS fifth edition. Radiographics 36(3): 623-639, 2016. PMID: 27082663. DOI: 10.1148/ rg.2016150178

22 Ambrogi V, Forcella D, Gatti A, Vanni G and Mineo TC: Transthoracic repair of Morgagni's hernia: a 20-year experience from open to video-assisted approach. Surg Endosc 21(4): $587-$ 591, 2007. PMID: 17180292. DOI: 10.1007/s00464-006-9017-7

23 Framarino-dei-Malatesta M, Derme M, Manzia TM, Iaria G, De Luca L, Fazzolari L, Napoli A, Berloco P, Patel T, Orlando G and Tisone G: Impact of mTOR-I on fertility and pregnancy: state of the art and review of the literature. Expert Rev Clin Immunol 9(8): 781-789, 2013. PMID: 23971756. DOI: 10.1586/ 1744666X.2013.824243

24 Noce A, Canale MP, Capria A, Rovella V, Tesauro M, Splendiani G, Annicchiarico-Petruzzelli M, Manzuoli M, Simonetti G and Di Daniele N: Coronary artery calcifications predict long term cardiovascular events in non diabetic Caucasian hemodialysis patients. Aging (Albany NY) 7(4): 269-279, 2015. PMID: 26131456. DOI: 10.18632/aging. 100740

25 Guarino M, Viganò L, Ponziani FR, Giannini EG, Lai Q, Morisco F and Special Interest Group on Hepatocellular carcinoma and new anti-HCV therapies" of the Italian Association for the Study of the Liver: Recurrence of hepatocellular carcinoma after direct acting antiviral treatment for hepatitis $\mathrm{C}$ virus infection: Literature review and risk analysis. Dig Liver Dis 50(11): 1105-1114, 2018. PMID: 30170908. DOI: 10.1016/j.dld.2018.08.001

26 Łuczyńska E, Heinze-Paluchowska S, Hendrick E, Dyczek S, Ryś J, Herman K, Blecharz P and Jakubowicz J: Comparison between breast MRI and contrast-enhanced spectral mammography. Med Sci Monit 21: 1358-1367, 2015. PMID: 25963880. DOI: $10.12659 /$ MSM.893018
27 Vanni G, Pellicciaro M, Materazzo M, Palombi L and Buonomo OC: Breast cancer diagnosis in Coronavirus-era: alert from Italy. Front Oncol 10: 938, 2020. PMID: 32574281. DOI: 10.3389/ fonc. 2020.00938

28 Vanni G, Materazzo M, Santori F, Pellicciaro M, Costesta M, Orsaria P, Cattadori F, Pistolese CA, Perretta T, Chiocchi M, Meucci R, Lamacchia F, Assogna M, Caspi J, Granai AV, DE Majo A, Chiaravalloti A, D'Angelillo MR, Barbarino R, Ingallinella S, Morando L, Dalli S, Portarena I, Altomare V, Tazzioli $G$ and Buonomo OC: The effect of Coronavirus (COVID-19) on breast cancer teamwork: a multicentric survey. In Vivo 34(3 Suppl): 1685-1694, 2020. PMID: 32503830. DOI: 10.21873/invivo.11962

29 Buonomo OC, Materazzo M, Pellicciaro M, Caspi J, Piccione E and Vanni G: Tor Vergata University-Hospital in the beginning of COVID-19-era: Experience and recommendation for breast cancer patients. In Vivo 34(3 Suppl): 1661-1665, 2020. PMID: 32503826. DOI: 10.21873/invivo.11958

30 Vanni G, Pellicciaro M, Materazzo M, Bruno V, Oldani C, Pistolese CA, Buonomo C, Caspi J, Gualtieri P, Chiaravalloti A, Palombi L, Piccione E and Buonomo OC: Lockdown of breast cancer screening for COVID-19: Possible scenario. In Vivo 34(5): 3047-3053, 2020. PMID: 32871851. DOI: 10.21873/invivo.12139

31 Sogani J, Morris EA, Kaplan JB, D’Alessio D, Goldman D, Moskowitz CS and Jochelson MS: Comparison of background parenchymal enhancement at contrast-enhanced spectral mammography and breast MR imaging. Radiology 282(1): 6373, 2017. PMID: 27379544. DOI: 10.1148/radiol.2016160284

32 King V, Brooks JD, Bernstein JL, Reiner AS, Pike MC and Morris EA: Background parenchymal enhancement at breast MR imaging and breast cancer risk. Radiology 260(1): 50-60, 2011. PMID: 21493794. DOI: 10.1148/radiol.11102156

Received December 18, 2021

Revised January 9, 2022

Accepted January 10, 2022 